\title{
Pseudomonas aeruginosa inhibits in-vitro Candida biofilm development
}

\author{
HMHN Bandara', JYY Yau', RM Watt' ${ }^{1}$ LJ Jin² and LP Samaranayake*1
}

\begin{abstract}
Background: Elucidation of the communal behavior of microbes in mixed species biofilms may have a major impact on understanding infectious diseases and for the therapeutics. Although, the structure and the properties of monospecies biofilms and their role in disease have been extensively studied during the last decade, the interactions within mixed biofilms consisting of bacteria and fungi such as Candida spp. have not been illustrated in depth. Hence, the aim of this study was to evaluate the interspecies interactions of Pseudomonas aeruginosa and six different species of Candida comprising C. albicans, C. glabrata, C. krusei, C. tropicalis, C. parapsilosis, and C. dubliniensis in dual species biofilm development.
\end{abstract}

Results: A significant reduction in colony forming units (CFU) of C. parapsilosis (90 min), C. albicans and C. tropicalis (90 min, $24 \mathrm{~h}$ and $48 \mathrm{~h}$ ), C. dubliniensis and C. glabrata, (24 h and $48 \mathrm{~h}$ ) was noted when co-cultured with P. aeruginosa in comparison to their monospecies counterparts $(P<0.05)$. A simultaneous significant reduction in $P$. aeruginosa numbers grown with C. albicans (90 min and 48 h), C. krusei (90 min, $24 \mathrm{~h}$ and 48 h),C. glabrata, (24 h and 48 h), and an elevation of $P$. aeruginosa numbers co-cultured with $C$. tropicalis $(48 \mathrm{~h}$ ) was noted $(\mathrm{P}<0.05)$. When data from all Candida spp. and P. aeruginosa were pooled, highly significant mutual inhibition of biofilm formation was noted (Candida P< 0.001 , P. aeruginosa P < 0.01). Scanning Electron Microscopy (SEM) and Confocal Laser Scanning Microscopy (CLSM) analyses confirmed scanty architecture in dual species biofilm in spite of dense colonization in monospecies counterparts.

Conclusions: $P$. aeruginosa and Candida in a dual species environment mutually suppress biofilm development, both quantitatively and qualitatively. These findings provide a foundation to clarify the molecular basis of bacterial-fungal interactions, and to understand the pathobiology of mixed bacterial-fungal infections.

\section{Background}

In most natural environments, microorganisms exist predominantly as biofilms rather than as free floating planktonic cells [1]. A biofilm can be defined as a complex functional community of one or more species of microbes encased in extra cellular polymeric substances and, attached to one another or to a solid surface [2]. Biofilms can be composed of a single microbial species or more commonly, mixed species such as bacteria and fungi $[3,4]$. Perhaps the most studied example of the biofilm in humans is the dental plaque[5]. Microorganisms in the biofilm characteristically display a phenotype that is markedly different from that of their free floating coun-

* Correspondence: lakshman@hkucc.hku.hk

1 Faculty of Dentistry, The University of Hong Kong, Oral Biosciences, 5/F, Prince Phillip Dental Hospital, 34, Hospital road, Sai Ying Pun, Hong Kong Full list of author information is available at the end of the article terparts [1]. For instance, they are resistant to antimicrobial agents in comparison to planktonic cells [6-8]. As more than $65 \%$ of biofilms with human microbial infections are caused by biofilms [5], there is an urgent need to understand biofilm behaviour.

The genus Candida comprises more than 150 pathogenic and nonpathogenic yeast species. Among these, $C$. albicans, C. tropicalis, C. parapsilosis, C. krusei, C. kefyr, C. glabrata and C. guillermondii are recognized as medically important pathogens [9]. C. albicans is the most prevalent yeast isolated from humans (47-75\%) followed by C. tropicalis (7\%), C. glabrata (7\%), C. krusei (5\%), C. parapsilosis $(<5 \%)$ and C. guillermondii $(<5 \%)$ [9]. Common Candidal habitats of humans include the gut, skin and mucosal surfaces, while one half of the human population carry Candida in their oral cavities[10]. 
Pseudomonas aeruginosa is an aerobic Gram-negative bacterium that causes community acquired infections, such as ulcerative keratitis, otitis externa, skin and soft tissue infections and, nosocomial infections including pneumonias, urinary tract infections, infections in surgical sites and burns [24,25]. Indeed, out of all nosocomial infections in different ethnic communities, $11-13.8 \%$ is found to be caused by $P$. aeruginosa [11-13]. United States Cystic Fibrosis Foundation Patients Registry (2004), has stated that $57.3 \%$ of all reported respiratory cultures contained $P$. aeruginosa indicating its important role in causing chronic and recurrent infections in cystic fibrotic patients [14]. Lee et al [15] have demonstrated that $P$. aeruginosa is the most commonly identified cause of septicemia in primary immunodeficiency and some $20 \%$ of bacteriaemia in acute leukemic patients [16,17]. Incidence of $P$. aeruginosa bacteriaemias in HIV affected patients is approximately 10 times higher than that of the normal population [18].

Pathogenic interactions between $C$. albicans and $P$. aeruginosa have recently been demonstrated by a number of groups $[19,20]$. The antifungal behaviour of $P$. aeruginosa against Candida spp. was first reported in early nineties by Kerr et al [20]. Subsequently others have shown that $P$. aeruginosa kills $C$. albicans by forming a dense film on fungal filaments, though, it neither binds nor kills the yeast-form of C. albicans [19]. Thein et al [21] have also reported that $P$. aeruginosa ATCC 27853 at a concentration gradient elicited a significant inhibition of Candida albicans biofilms.

Although, the structure and the properties of monospecies biofilms and their role in disease have been extensively studied during the last decade [22,23], the interactions within mixed biofilms consisting of bacteria and fungi including Candida spp. have not been studied in depth. Furthermore, the majority of the previous studies on interactions between Candida and bacteria in mixed biofilms have focused on C. albicans and there are only a few studies on non-albicans Candida spp. biofilms in a mixed species environment. Hence, the aims of this study were to evaluate the interactions of a reference laboratory strain of $P$. aeruginosa and six different Candida species, C. albicans, C. glabrata, C. tropicalis, C. parapsilosis, C. dubliniensis, and C. krusei in a dual species biofilms environment over a period of 2 days by both quantitative assays (Colony Forming Unit assay - CFU) and, qualitative evaluations using Scanning Electron Microscopy (SEM) and Confocal Laser Scanning microscopy (CLSM).

\section{Results}

Candida and $\mathrm{P}$. aeruginosa dual species biofilm growth

After 90 min of biofilm development with $P$. aeruginosa, a significant, $57-88 \%$, reduction in Candida counts was noted for $C$. albicans (57\%, $\mathrm{P}=0.005), C$. dubliniensis (69\%, $\mathrm{P}<0.001), C$. tropicalis $(18 \%, \mathrm{P}=0.010)$ and $C$. parapsilosis $(74 \%, \mathrm{P}=0.030)$ while $P$. aeruginosa did not impart such an effect on C. glabrata and C. krusei compared with the controls (Table 1). Conversely, after 90 min, a significant reduction in CFU of $P$ aeruginosa was observed in the presence of C. albicans $(81 \%, \mathrm{P}=0.002)$ C. krusei $(62 \%, \mathrm{P}=0.002)$ but not with the other four Candida species (Table 1).

However, after prolonged incubation for 24 hours, a significant, 58-91\% reduction in the counts of $C$. albicans (67\%, $\mathrm{P}<0.001)$, C. tropicalis $(88 \%, \mathrm{P}<0.001)$ C. dubliniensis $(91 \%, \mathrm{P}<0.001)$ and C. glabrata $(58 \%, \mathrm{P}=0.024)$ was noted in dual species biofilms with $P$. aeruginosa (Table 1) although $C$. krusei and $C$. parapsilosis counts were unaffected in comparison to the monospecies controls. On the other hand, mean CFU of P. aeruginosa decreased significantly in the presence of C. krusei (41\%, $\mathrm{P}=0.022), C$. dubliniensis $(48 \%, \mathrm{P}=0.003)$ and $C$. glabrata $(83 \%, \mathrm{P}<0.001)$ after $24 \mathrm{~h}$, while the other three Candida species had no significant effect on $P$. aeruginosa numbers at this time point (Table 1).

Most remarkable results were observed on further incubation for 48 hours, C. albicans (99\%, $\mathrm{P}<0.001), C$. tropicalis $(100 \%, \mathrm{P}<0.001)$ and C. glabrata $(100 \%, \mathrm{P}<$ 0.001 ) growth was almost totally suppressed in dual species biofilms with $P$. aeruginosa while the remaining Candida species were unaffected (Table 1). Simultaneously the mean CFU of $P$. aeruginosa decreased in co cultures of C. albicans (32\%, P = 0.009) C. krusei (48\%, $\mathrm{P}=0.010)$, and C. glabrata (78\%, $\mathrm{P}<0.001)$. Conversely, P. aeruginosa counts significantly increased in the presence of $C$. tropicalis $(72 \%, \mathrm{P}=0.002)$. Such an effect was not seen after $48 \mathrm{~h}$ with the two remaining Candida species, $C$. dubliniensis and C. parapsilosis (Table 1).

Despite these variable results, at different time intervals, when data from all Candida spp. were pooled and analyzed, a highly significant inhibition of Candida biofilm formation by $P$. aeruginosa $(\mathrm{P}<0.001)$ and a simultaneous significant inhibition of $P$. aeruginosa biofilm development by Candida at all three time intervals $(\mathrm{P}<$ 0.01 ) was noted.

\section{Confocal laser scanning microscopy}

CLSM with Live and Dead stain confirmed, in general, that Candida spp. and P. aeruginosa have mutually suppressive effects on each other at every stage of biofilm formation, in comparison to their monospecies counterparts. CLSM showed a reduction in both Candida and $P$. aeruginosa cells that were adherent after $90 \mathrm{~min}$, confirming the data from CFU assay. Few dead C. albicans cells were also visible (Figure 1A, B and 1C).

In $24 \mathrm{~h}$-dual species biofilms, mutual suppression of $C$. dubliniensis and $P$. aeruginosa was clearly seen, confirm- 
Table 1: The mean CFU counts $( \pm$ SD) of Candida spp. and $P$. aeruginosa from both monospecies and dual species biofilms at $90 \mathrm{~min}, 24 \mathrm{~h}$ and $48 \mathrm{~h}$.

\begin{tabular}{|c|c|c|c|c|c|c|c|}
\hline & \multirow{2}{*}{$\begin{array}{c}\text { Time } \\
\text { interval }\end{array}$} & \multicolumn{2}{|c|}{ Candida CFU (106) \pm SD } & \multirow[t]{2}{*}{ P value } & \multicolumn{2}{|c|}{ P. aeruginosa CFU (106) \pm SD } & \multirow[t]{2}{*}{ P value } \\
\hline & & Control (MSB) & Test (DSB) & & Control (MSB) & Test (DSB) & \\
\hline \multirow{3}{*}{$\begin{array}{l}\text { Candida } \\
\text { albicans }\end{array}$} & $90 \min$ & $12.60 \pm 2.19$ & $5.29 \pm 1.52$ & 0.005 & $3.44 \pm 2.20$ & $0.66 \pm 0.69$ & 0.002 \\
\hline & $24 \mathrm{~h}$ & $15.22 \pm 3.31$ & $5.00 \pm 2.60$ & $<0.001$ & $876.89 \pm 206.39$ & $719.56 \pm 266.53$ & 0.200 \\
\hline & $48 \mathrm{~h}$ & $31.89 \pm 6.60$ & $0.22 \pm 0.44$ & $<0.001$ & $1358.89 \pm 323.59$ & $922.22 \pm 186.60$ & 0.009 \\
\hline \multirow[t]{3}{*}{ Candida krusei } & $90 \min$ & $2.43 \pm 1.46$ & $2.71 \pm 0.66$ & 0.352 & $7.32 \pm 3.82$ & $2.78 \pm 1.29$ & 0.003 \\
\hline & $24 \mathrm{~h}$ & $3.39 \pm 2.00$ & $2.49 \pm 0.73$ & 0.301 & $987.78 \pm 341.79$ & $583.33 \pm 218.92$ & 0.022 \\
\hline & $48 \mathrm{~h}$ & $0.09 \pm 0.14$ & $0.22 \pm 0.44$ & 0.867 & $140.00 \pm 48.73$ & $73.33 \pm 35.71$ & 0.010 \\
\hline \multirow{3}{*}{$\begin{array}{l}\text { Candida } \\
\text { tropicalis }\end{array}$} & $90 \min$ & $9.81 \pm 3.05$ & $3.87 \pm 2.29$ & 0.004 & $1.42 \pm 1.25$ & $2.26 \pm 0.71$ & 0.070 \\
\hline & $24 \mathrm{~h}$ & $27.67 \pm 5.92$ & $3.44 \pm 1.59$ & $<0.001$ & $431.11 \pm 66.23$ & $471.11 \pm 162.90$ & 0.534 \\
\hline & $48 \mathrm{~h}$ & $4.22 \pm 2.05$ & $0.00 \pm 0.00$ & $<0.001$ & $98.89 \pm 75.74$ & $351.11 \pm 162.51$ & 0.002 \\
\hline \multirow{3}{*}{$\begin{array}{c}\text { Candida } \\
\text { parapsilosis }\end{array}$} & $90 \mathrm{~min}$ & $10.60 \pm 6.71$ & $1.26 \pm 1.34$ & $<0.001$ & $4.87 \pm 1.66$ & $3.83 \pm 2.31$ & 0.228 \\
\hline & $24 \mathrm{~h}$ & $2.11 \pm 2.32$ & $0.78 \pm 0.44$ & 0.364 & $412.22 \pm 208.55$ & $277.78 \pm 162.69$ & 0.121 \\
\hline & $48 \mathrm{~h}$ & $0.89 \pm 0.60$ & $0.44 \pm 0.73$ & 0.120 & $183.33 \pm 69.64$ & $179.56 \pm 50.02$ & 0.859 \\
\hline \multirow{3}{*}{$\begin{array}{l}\text { Candida } \\
\text { glabrata }\end{array}$} & $90 \mathrm{~min}$ & $10.81 \pm 2.90$ & $10.12 \pm 3.97$ & 0.659 & $9.91 \pm 9.01$ & $8.17 \pm 5.03$ & 0.691 \\
\hline & $24 \mathrm{~h}$ & $35.78 \pm 21.72$ & $15.00 \pm 21.08$ & 0.024 & $328.89 \pm 88.94$ & $56.67 \pm 15.81$ & $<0.001$ \\
\hline & $48 \mathrm{~h}$ & $28.22 \pm 17.14$ & $0.11 \pm 0.33$ & $<0.001$ & $128.89 \pm 69.54$ & $28.89 \pm 17.64$ & $<0.001$ \\
\hline \multirow{3}{*}{$\begin{array}{c}\text { Candida } \\
\text { dubliniensis }\end{array}$} & $90 \mathrm{~min}$ & $9.34 \pm 3.21$ & $2.94 \pm 1.50$ & $<0.001$ & $9.83 \pm 2.33$ & $6.51 \pm 4.35$ & 0.070 \\
\hline & $24 \mathrm{~h}$ & $5.81 \pm 2.46$ & $0.54 \pm 0.88$ & $<0.001$ & $878.89 \pm 286.07$ & $461.11 \pm 142.78$ & 0.003 \\
\hline & $48 \mathrm{~h}$ & $0.00 \pm 0.00$ & $0.00 \pm 0.00$ & 1.000 & $97.78 \pm 48.16$ & $52.22 \pm 50.94$ & 0.056 \\
\hline
\end{tabular}

$\mathrm{P}<0.05$ was considered statistically significant. Significant differences are shown in bold text.

ing CFU data. Thus, sparsely developed C. dubliniensis biofilm was seen with few dead cells in contrast to its dense monospecies biofilm, while $P$. aeruginosa numbers were greatly reduced compared to its monospecies counterpart (Figure 1D, E and 1F).

Similarly, after $48 \mathrm{~h}$, sparsely distributed C. tropicalis blastospores were noted in dual species biofilms with few, scattered $P$. aeruginosa cells and a scant biofilm once again confirming the aforementioned quantitative CFU findings. Some dead cells and cellular debris were also observed compared to dense monospecies biofilm growth of $C$. tropicalis control (figure $1 \mathrm{G}, \mathrm{H}$ and 1I).

\section{Scanning Electron Microscopy}

Although species specific growth variations could be noted, in general, single species biofilms of all Candida species demonstrated profuse growth and dense colonization of the substrate on SEM observation (Figure 2). After $90 \mathrm{~min}$, i.e. adhesion phase, the control monospecies Candida and P. aeruginosa cells were seen welladherent and uniformly distributed on the polystyrene surface. Yeast blastospores were seen aggregated either in pairs or clumps with some budding yeasts. During $24 \mathrm{~h}$ of initial colonization phase, monospecies biofilms of both Candida and P. aeruginosa showed increased numbers of 
(A)

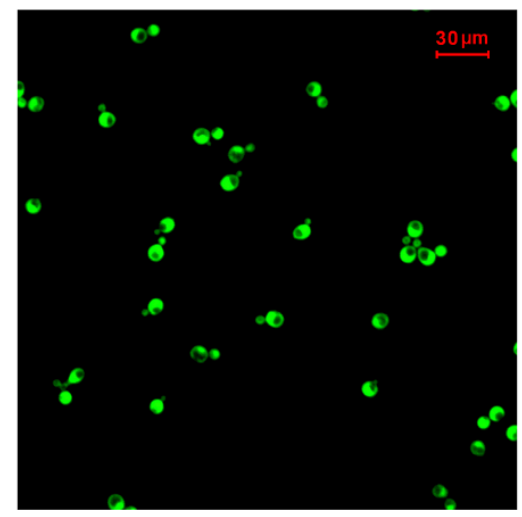

(D)

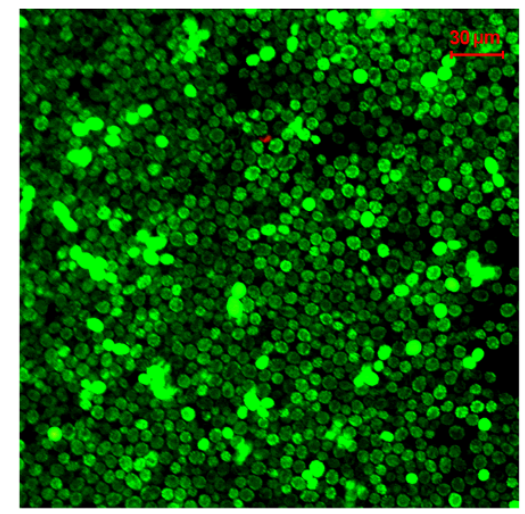

(G)

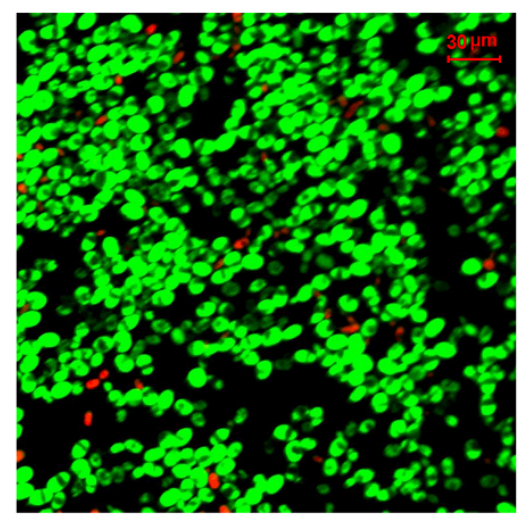

(B)

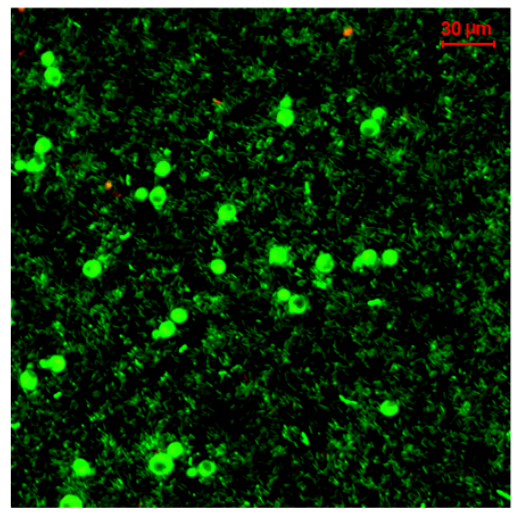

(E)

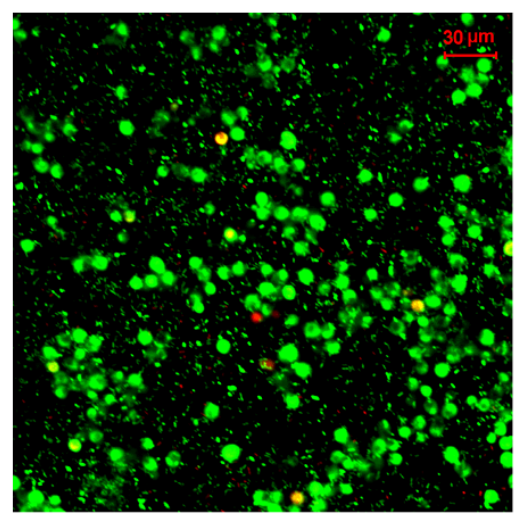

$(\mathrm{H})$

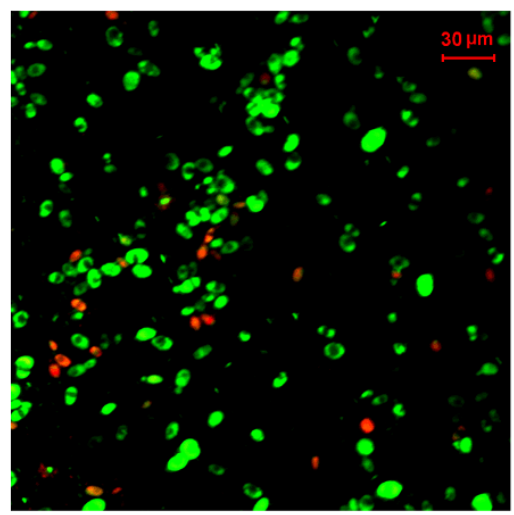

(C)

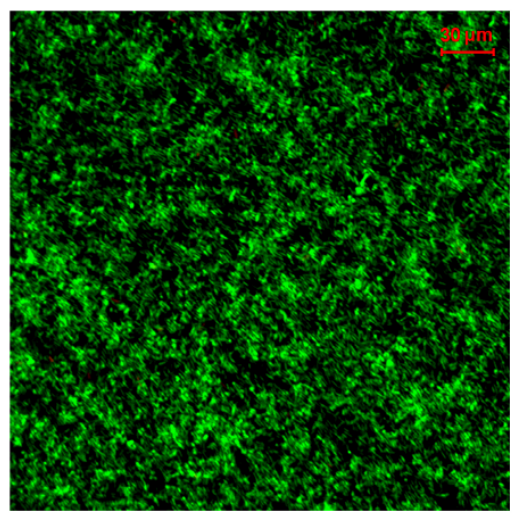

(F)

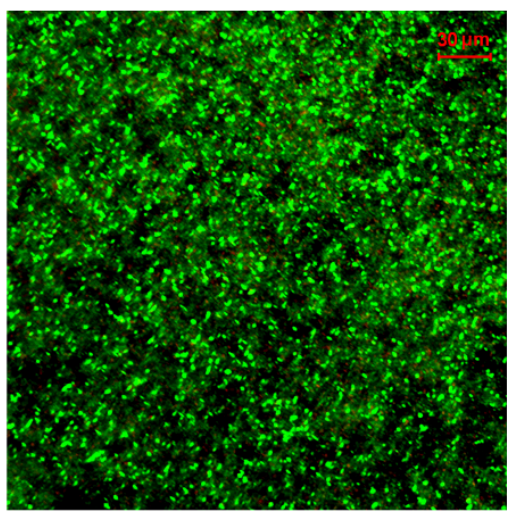

(I)

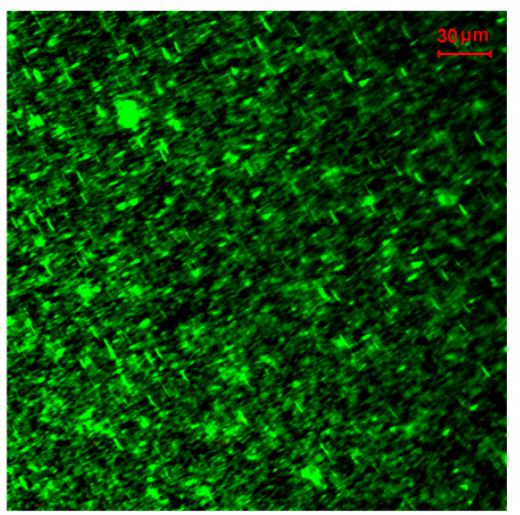

Figure 1 CLSM images of monospecies (Candida spp. or P. aeruginosa) and dual species (Candida spp. and P. aeruginosa) biofilms. (A). Adhesion of C. albicans for $90 \mathrm{~min}$, (B). Adhesion of C. albicans and P. aeruginosa for $90 \mathrm{~min}$, (C). Adhesion of $P$. aeruginosa for 90 min. Note the mutual inhibition of adhesion of both pathogens in dual species environment. (D) Initial colonization of C. dubliniensis for $24 \mathrm{~h}$ (E). Initial colonization of C. dubliniensis and $P$. aeruginosa for $24 \mathrm{~h}$, (F). Initial colonization of $P$. aeruginosa for $24 \mathrm{~h}$. Note the impaired biofilm formation after $24 \mathrm{~h}$ in the dual species biofilm due to mutual inhibition of these organisms. (G) Maturation of C. tropicalis for $48 \mathrm{~h}$, (H). Maturation of C. tropicalis and P. aeruginosa for $48 \mathrm{~h}$, (I). maturation of $P$. aeruginosa for $48 \mathrm{~h}$. Note the altered and scant biofilm maturation in dual species biofilm as a result of mutual inhibition of $C$. tropicalis and $P$. aeruginosa. 
cellular layers with recognizable extracellular matrix. After $48 \mathrm{~h}$, the single species biofilms of both pathogens were relatively thick and multilayered, although the extracellular matrix was scarcely visible.

However, on visual examination by SEM, dual species biofilms demonstrated reduction of yeast blastospores at each stage of biofilm formation compared to their monospecies counterparts. Specially in the maturation stage at $48 \mathrm{~h}$, this reduction was marked and recognizable. The former biofilms were also less dense than the monospecies controls, and demonstrated few layers of cells, profuse cellular debris, together with degrading and morphologically altered yeast cells. Interestingly, most of the bacteria were seen attached to the blastospores (figure $2 \mathrm{E}$ and $2 \mathrm{H})$. Bacterial density varied in the presence of different Candida species at different time intervals. In general, $P$. aeruginosa distribution was scanty and nondescript in the dual species environment (Figure 2B, E and $2 \mathrm{H})$.

Quantitatively, smaller numbers of clumped C. albicans, together with some degrading blastospores, were observed with $P$. aeruginosa at the end of the adhesion phase, and the latter was also lesser in number compared to the monospecies variant (Figure 2A, B and 2C). A thin, scant biofilm, formed by a lesser numbers of morphologically altered C. glabrata was noted after initial colonization (Figure 2C, D and 2E). Furthermore, a few, morphologically altered blastospores of C. tropicalis were visible in mature dual species biofilm with $P$. aeruginosa at $48 \mathrm{~h}$. In contrast, $P$. aeruginosa demonstrated thicker biofilms in the presence of $C$. tropicalis, compared to its mature monospecies variant (Figure 2G, H and 2I).

\section{Discussion}

Candida and P. aeruginosa are major pathogens of device-associated nosocomial infections for virtually all types of indwelling devices [24]. It has also been stated that, the coexistence of Pseudomonas spp. and C. albicans in elderly is a potential indicator of high risk for pneumonia [25]. Recent experimental studies have identified similarities in environmental factors such as its physical and chemical nature where $P$. aeruginosa and $C$. albicans coexist [26]. As a result, these two microorganisms have become obvious candidates and models for the study of biofilm infections in order to develop potential methods for the control of device-associated nosocomial infections[24].

The principle aim of this study was to evaluate the qualitative and quantitative effects of $P$. aeruginosa on various stages of in-vitro biofilm formation of six different Candida species. Our results indicate that both Candida and $P$. aeruginosa mutually inhibit biofilm development to varying degrees at different stages of biofilm formation. However, the most important conclusion of our study is the ability of $P$. aeruginosa to almost totally inhibit $C$. albicans, C. glabrata and C. tropicalis in $48 \mathrm{~h}$ biofilms.

Using a CFU assay, we report here for the first time, the quantitative effect of $P$. aeruginosa on biofilm formation of six different Candida species in a time dependant manner. Our results indicate that $P$. aeruginosa had significant inhibitory effects on several Candida spp. such as, C. albicans, C. dubliniensis, C. tropicalis, and $C$. parapsilosis. In contrast, El-Azizi [27] found that Pseudomonas had no significant effect on C. albicans adhesion and biofilm growth, regardless of adding preformed Pseudomonas biofilms to C. albicans or vice versa. As there appeared to be differences in the mode of attachment of $P$. aeruginosa to yeast form of $C$. albicans or its filamentous form [28], mixed biofilm development between these two organisms could be a function of these characteristics.

Thein et al [21] from our group reported that, on prolong incubation for 2 days, P. aeruginosa ATCC 27853 at a concentration gradient, elicited a significant inhibition of C. albicans biofilm with a mean reduction in the number of viable Candidal cells ranging from $38 \%$ to $81 \%$. Our results extend their work further and indicate that $P$. aeruginosa suppresses several other Candida species on incubation for upto two days, for instance, C. dubliniensis at $24 \mathrm{~h}$ and,C. albicans, C. glabrata and C. tropicalis both at $24 \mathrm{~h}$ and $48 \mathrm{~h}$. In this context, Kaleli et al [29] investigated the anticandidial activity of 44 strains of $P$. aeruginosa, isolated from a number of specimens of intensive care patients, against four Candida species (C. albicans, C. tropicalis, C. parapsilosis and C. krusei) by a cross streak assay and subcutaneous injections of both bacterial and fungal suspensions into mice. They found that all Pseudomonas strains tested inhibited all four Candida species to varying degrees. C. albicans and C. krusei were the most inhibited while C. tropicalis were the least [29]. In contrast, our data show that the most significant inhibition elicited by $P$. aeruginosa was $C$. albicans and $C$. tropicalis while, the least was C. krusei. Grillot et al [30] observed complete or partial inhibition of C. albicans, $C$. tropicalis, C. parapsilosis and C. glabrata by P. aeruginosa in pure and mixed blood cultures using in-vitro yeast inhibition assays and suggested that preclusion of yeast recovery from blood cultures in mixed infections, such as polymicrobial septicemia, may be due to suppression of yeast by $P$. aeruginosa. In another study Kerr [20] demonstrated that nine Candida species, out of eleven tested, including C. krusei, C. kefyr, C. guillermondii, C. tropicalis, C. lusitaniae, C. parapsilosis, C. pseudotropicalis, C. albicans and Torulopsis glabrata were suppressed by $P$. aeruginosa. This in-vitro susceptibility test was performed with ten different strains of $P$. aeruginosa obtained from the sputum of three patients. Moreover, $C$. albicans was the most susceptible to growth inhibition 


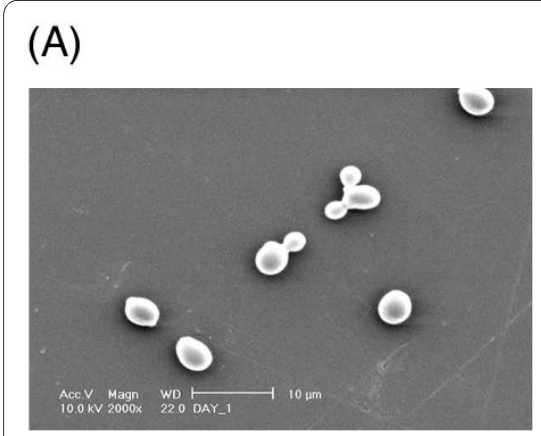

(D)

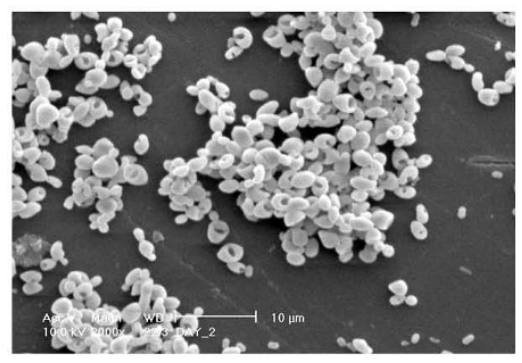

(G)

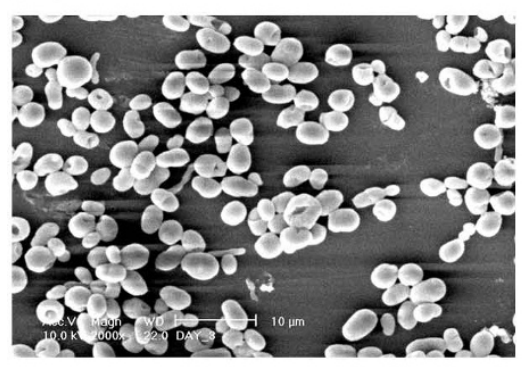

(B)

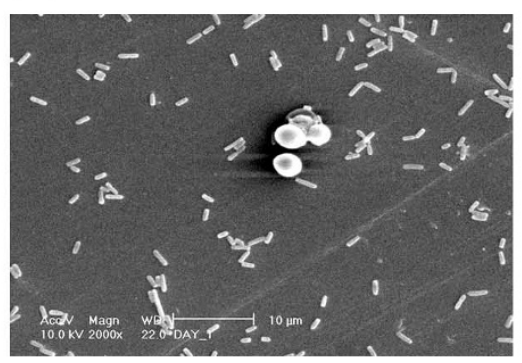

(E)

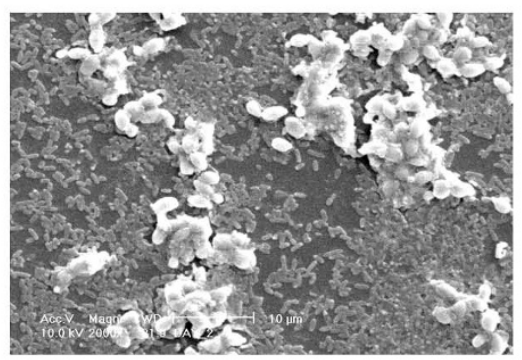

(C)

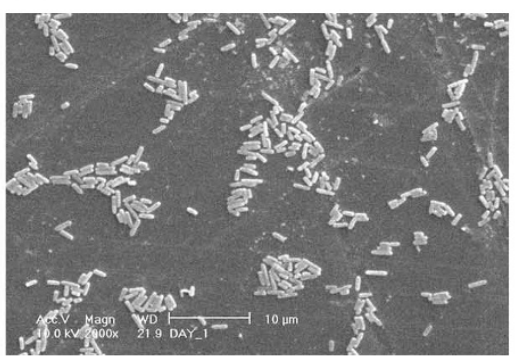

(F)

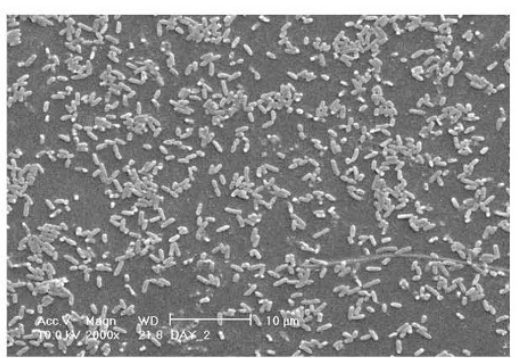

(H)

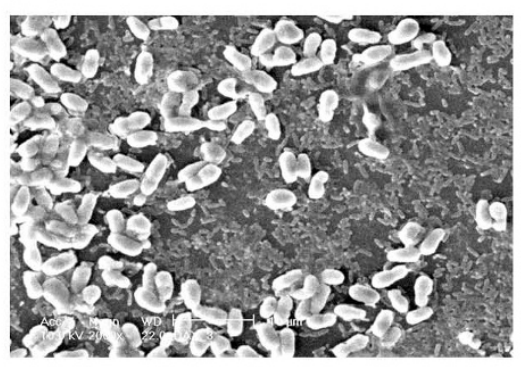

(l)

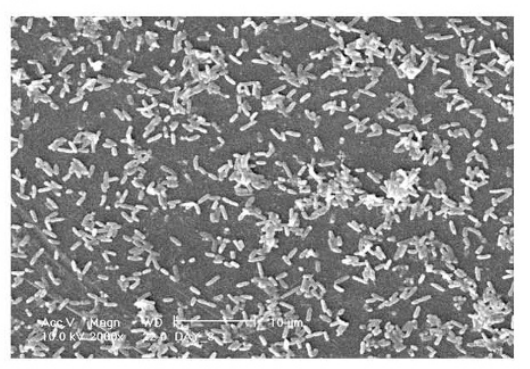

Figure 2 SEM images of monospecies (Candida spp. or P. aeruginosa) and dual species (Candida spp. and P. aeruginosa) biofilms. (A). Adhesion of C. albicans for $90 \mathrm{~min}$, (B). Adhesion of C. albicans and P. aeruginosa for $90 \mathrm{~min}$, (C). Adhesion of P. aeruginosa for $90 \mathrm{~min}$. Note that there are few C. albicans blastospores with some degrading cells and few cells of $P$. aeruginosa in dual species biofilm in compared to monospecies counterparts. (D) Initial colonization of C. glabrata for $24 \mathrm{~h}$ (E). Initial colonization of C. glabrata and P. aeruginosa for $24 \mathrm{~h}$, (F). Initial colonization of $P$. aeruginosa for $24 \mathrm{~h}$. Note that C. glabrata is less in number with altered morphology while thin and scant biofilm was formed in the presence of $P$. aeruginosa. (G) Maturation of C. tropicalis for $48 \mathrm{~h}$, (H). Maturation of C. tropicalis and P. aeruginosa for $48 \mathrm{~h}$, (I). Maturation of P. aeruginosa for $48 \mathrm{~h}$. Note the reduction in number and altered morphology of C. tropicalis in dual species biofilm.

followed by C. guillermondii and T. glabrata. Hockey et al [31], using an in-vitro model, studied the interactions of six different bacteria including $P$. aeruginosa and three pathogenic Candida species (C. albicans, C. tropicalis, and T. glabrata). The results of this study indicated that all three Candida species were suppressed by $P$. aeruginosa and Klebsiella pneumoniae in culture media. They further explained that this inhibition could be due to nutritional depletion and secretion of bacterial toxins. Interestingly, our results in general, concur with the foregoing findings as we too noted a significant inhibitory effect of P. aeruginosa on C. albicans, C. tropicalis and C. parapsilosis at different stages of their biofilm development. However, it should be emphasized that all of the foregoing studies were done in mixed culture media and our results are derived from a biofilm model.

In addition, as our study was bidirectional, we noted that some of the Candida species also suppressed $P$. aeruginosa during adhesion, initial colonization and maturation in dual species environment. Particularly, C. albicans at $90 \mathrm{~min}, \mathrm{C}$. dubliniensis at $24 \mathrm{~h}, \mathrm{C}$. albicans, C. 
krusei, and C. glabrata at both 24 and $48 \mathrm{~h}$ and C. tropicalis at $48 \mathrm{~h}$.

Therefore, our results further authenticate the mutual inhibition and aggregation of certain Candida spp. and $P$. aeruginosa. Further works with multiple strains of Candida from different species are requested to confirm the species specificity of these findings.

Ultrastructural views of both monospecies and dual species biofilms confirmed the results obtained from quantitative assays. Basically, all monospecies biofilms of both Candida and P. aeruginosa demonstrated a well organized biofilm structure where yeasts were uniformly distributed with minimal amounts of extracellular substance, dead cells and cellular debris. The mature monospecies biofilms showed a characteristically thick layered structure. In contrast, dual species biofilms consisted of less dense Candida and $P$. aeruginosa growth, larger numbers of clumped cells, dead cells and cellular debris demonstrating the mutual inhibitory effect of these two pathogens in a dual species environment.

\section{Conclusions}

In conclusion, this study, principally focused on the interactions of Candida spp. and $P$. aeruginosa during different stages of biofilm development, indicates the latter pathogens have significant mutual growth inhibitory effect at various stages of biofilm development in a dual species environment. It is also evident that there are species specific variations of this modulatory effect. Further work is necessary to clarify the molecular basis of these bacterial-fungal interactions, and to understand the pathobiology of mixed bacterial-fungal infections.

\section{Methods}

\section{Experimental design}

The study comprised a series of experiments to evaluate the combined effect of each of the aforementioned six Candida spp. and P. aeruginosa on their biofilm formation, quantitatively with CFU assay and qualitatively with CLSM and SEM, at three different time intervals, $90 \mathrm{~min}$, $24 \mathrm{~h}$ and $48 \mathrm{~h}$.

\section{Microorganisms}

The following Reference laboratory strains of both Candida and $P$. aeruginosa were used, Candida albicans ATCC 90028, Candida glabrata ATCC 90030, Candida tropicalis ATCC 13803, Candida parapsilosis ATCC 22019, Candida krusei ATCC 6258, Candida dubliniensis MYA 646 and Pseudomonas aeruginosa ATCC 27853. The identity of each organism was confirmed with the commercially available API $32 \mathrm{C}$ (for Candida strains) and API $20 \mathrm{E}$ (for $P$. aeruginosa) identification systems (Biomérieux, Mercy I'Etoile, France). All isolates were stored in multiple aliquots at $-20^{\circ} \mathrm{C}$, after confirming their purity.

\section{Growth media}

Sabouraud Dextrose Agar (SDA), Yeast Nitrogen Base (YNB) solution supplemented with $100 \mathrm{mM}$ glucose were used for culturing Candida species while, Blood agar, MacConkey agar and Tryptic Soy Broth (TSB) were utilized for $P$. aeruginosa culture.

\section{Microbial inocula}

Prior to each experiment, Candida spp. and P. aeruginosa were subcultured on SDA and blood agar, respectively for $18 \mathrm{~h}$ at $37^{\circ} \mathrm{C}$. A loopful of the overnight Candida growth was inoculated into YNB medium, $P$. aeruginosa into TSB medium and, incubated for $18 \mathrm{~h}$ in an orbital shaker $(75$ $\mathrm{rpm})$ at $37^{\circ} \mathrm{C}$. The resulting cells were harvested, washed twice in Phosphate Buffered Saline (PBS, pH 7.2) and resuspended. Concentrations of Candida spp. and $P$. aeruginosa were adjusted $1 \times 10^{7}$ cells $/ \mathrm{mL}$ by spectrophotometry and confirmed by hemocytometric counting.

\section{Biofilm Formation}

Candida biofilms were developed as described by Jin et al [32] with some modifications. Commercially available pre-sterilized, polystyrene, flat bottom 96 -well microtiter plates (IWAKI, Tokyo, Japan) were used. At first, $100 \mu \mathrm{L}$ of standard cell suspensions of Candida spp. and P. aeruginosa $\left(10^{7}\right.$ organisms $/ \mathrm{mL}, 1: 1$ ratio) were prepared and transferred into selected wells of a microtiter plate, and incubated for $90 \mathrm{~min}$ at $37^{\circ} \mathrm{C}$ in an orbital shaker at 75 rpm to promote microbial adherence to the surface of the wells. Hundred microliters of monospecies controls of both Candida spp. and $P$. aeruginosa were inoculated in an identical fashion. After the adhesion phase, the cell suspensions were aspirated and each well was washed twice with PBS to remove loosely adherent cells. A total of $200 \mu \mathrm{L}$ of TSB was transferred to each well and the plate reincubated for $24 \mathrm{~h}$ and for $48 \mathrm{~h}$, and wells washed twice and thrice at respective time intervals with PBS to eliminate traces of TSB. The bacterial/fungal interactions were studied at $90 \mathrm{~min}, 24 \mathrm{~h}$, and $48 \mathrm{~h}$ time intervals as follows.

\section{Quantitative analyses}

\section{Spiral plating and colony forming units assay (CFU)}

At the end of the adhesion (90 min), colonization (24 h) and maturation $(48 \mathrm{~h}$ ) phases, $100 \mu \mathrm{L}$ of PBS was transferred into each well and the biofilm mass was meticulously scraped off the well-wall using a sterile scalpel [32]. The resulting suspension containing the detached biofilm cells was gently vortexed for $1 \mathrm{~min}$ to disrupt the aggregates, serially diluted, and inoculated by a spiral plater on SDA for Candida spp. and, on MacConkey agar for $P$. 
aeruginosa. The resulting CFU of yeasts and bacteria were quantified after $48 \mathrm{~h}$ incubation at $37^{\circ} \mathrm{C}$. Each assay was carried out in triplicate at three different points in time.

\section{Qualitative analyses}

Confocal Laser Scanning Microscopy (CLSM) [33] and Scanning Electron microscopy (SEM) were used to observe the ultrastructure of Candida and P. aeruginosa biofilms.

\section{Confocal Laser Scanning Microscopy}

Commercially available presteriled flat bottom six well plates (Iwaki, Japan) and presteriled plastic coupons (Thermanox plastic cover slips, Nulge Nunc International, Rochester, NY, USA) [34] were used to prepare biofilms as described above. Presteriled coupons were placed in wells of a 6-well plate, suspensions of monospecies or dual species added and the plate incubated for 90 min (the adhesion phase) in an orbital shaker (75 rpm) at $37^{\circ} \mathrm{C}$. Thereafter, the supernatant was removed, washed twice with PBS, fresh TSB added and incubated for 24 hours (initial colonization) or 48 hours (maturation) under same environmental conditions. At the end of each time interval, the prewashed coupons were stained with Live and Dead stain (Live/Dead BacLight Bacterial Viability kit, Invitrogen, Eugene, USA). The biofilm architecture was then analyzed by fluorescent microscopy (using Confocal Laser Scanning Microscope).

\section{Scanning Electron Microscopy}

For SEM, we developed single species biofilms (Candida alone and $P$. aeruginosa alone) as well as Candida and $P$. aeruginosa mixed biofilms on custom made, tissue culture treated, polystyrene coupons as described above. At $90 \mathrm{~min}, 24 \mathrm{~h}, 48 \mathrm{~h}$, selected coupons were removed from the wells, washed twice with PBS and placed in 1\% osmium tetroxide for $1 \mathrm{~h}$. Samples were subsequently washed in distilled water, dehydrated in increasing concentrations of ethanol ( $70 \%$ for $10 \mathrm{~min}$, $95 \%$ for $10 \mathrm{~min}$, and $100 \%$ for $20 \mathrm{~min}$ ), and air dried in a desiccator prior to sputter coating with gold. Then the specimens were mounted on aluminium stubs, with copper tape, coated with gold under low-pressure with an ion sputter coater (JEOL JFC1 100: JEOL, Tokyo, Japan). The surface topographies of the biofilm were visualized with a scanning electron microscope (Philip XL30CP) in high-vacuum mode at $10 \mathrm{kV}$, and the images processed.

\section{Statistical analysis}

Statistical analysis was performed using SPSS software (version 16.0). Mann--Whitney U test was performed to compare the significant differences between control and each test sample of the bacterial/Candidal biofilm. Data from all Candida spp. and $P$. aeruginosa analyses at different time points were pooled, and evaluated using Wil- coxon matched-pairs test. A P-value of $<0.05$ was considered statistically significant.

\section{Abbreviations}

CFU: Colony Forming Unit; SEM: Scanning Electron Microscopy; CLSM: Confocal Laser Scanning Microscopy; SDA: Sabouraud Dextrose Agar; YNB: Yeast Nitrogen Base; TSB: Tryptic Soy Broth; PBS: Phosphate Buffered Saline.

\section{Authors' contributions}

LPS, LJ, RMW and HMHNB conceived this research. HMHNB and JYYY designed and performed the experiments. HMHNB, LPS, LJ contributed in data analysis and interpretation. HMHNB drafted the manuscript and it was reviewed by LPS, LJJ, RMW and JYYY. All authors read and approved the final manuscript.

\section{Acknowledgements}

Authors would like to acknowledge Dr. Zaw Moe Thein for his advice. This study was supported by the grant of CERG HKU 7624/06M of The University of Hong Kong

\section{Author Details}

${ }^{1}$ Faculty of Dentistry, The University of Hong Kong, Oral Biosciences, 5/F, Prince Phillip Dental Hospital, 34, Hospital road, Sai Ying Pun, Hong Kong and 2Faculty of Dentistry, The University of Hong Kong, The Department of Periodontology, 3/F, Prince Phillip Dental Hospital, 34, Hospital road, Sai Ying Pun, Hong Kong

Received: 15 September 2009 Accepted: 25 April 2010

Published: 25 April 2010

\section{References}

1. Douglas LJ: Candida biofilms and their role in infection. Trends Microbiol 2003, 11:30-36

2. Samaranayake LP: Essential microbiology for dentistry. 3rd edition. Edinburgh: Churchill Livingstone; 2006.

3. Costerton JW, Stewart PS, Greenberg EP: Bacterial biofilms: a common cause of persistent infections. Science 1999, 284(5418):1318-1322.

4. Jenkinson HF, Douglas LJ: Interactions between Candida species and and bacteria in mixed infections. In Polymicrobial diseases Edited by: Brogden KA, Guthmiller JM. ASM Press; 2002:357-373.

5. Potera C: Forging a link between biofilms and disease. Science 1999 , 283(5409):1837-839.

6. Costerton JW, Lewandowski Z, Caldwell DE, Korber DR, Lappin-Scott HM: Microbial biofilms. Annu Rev Microbiol 1995, 49:711-745.

7. O'Toole G, Kaplan HB, Kolter R: Biofilm formation as microbial development. Annu Rev Microbiol 2000, 54:49-79.

8. Mah TF, O'Toole GA: Mechanisms of biofilm resistance to antimicrobial agents. Trends Microbio/ 2001, 9(1):34-39.

9. Odds FC: Candida and Candidosis. 2nd edition. London: Bailliere Tindall; 1988

10. Samaranayake LP, MacFarlane TW: Oral candidosis. London: Wright; 1990.

11. Pittet $D$, Harbarth $S$, Ruef C, Francioli P, Sudre P, Petignat C, Trampuz A, Widmer A: Prevalence and risk factors for nosocomial infections in four university hospitals in Switzerland. Infect Control Hosp Epidemiol 1999, 20(1):37-42

12. Lizioli A, Privitera G, Alliata E, Antonietta Banfi EM, Boselli L, Panceri ML, Perna MC, Porretta AD, Santini MG, Carreri V: Prevalence of nosocomial infections in Italy: result from the Lombardy survey in 2000. J Hosp Infect 2003, 54(2):141-148.

13. Kim JM, Park ES, Jeong JS, Kim KM, Oh HS, Yoon SW, Chang HS, Chang KH, Lee SI, Lee MS, et al:: Multicenter surveillance study for nosocomial infections in major hospitals in Korea. Nosocomial Infection Surveillance Committee of the Korean Society for Nosocomia Infection Control. Am J Infect Control 2000, 28(6):454-458.

14. Driscoll JA, Brody SL, Kollef MH: The epidemiology, pathogenesis and treatment of Pseudomonas aeruginosa infections. Drugs 2007, 67(3):351-368

15. Lee WI, Jaing TH, Hsieh MY, Kuo ML, Lin SJ, Huang JL: Distribution, infections, treatments and molecular analysis in a large cohort of 
patients with primary immunodeficiency diseases (PIDs) in Taiwan. $J$ Clin Immunol 2006, 26(3):274-283.

16. Chatzinikolaou I, Abi-Said D, Bodey GP, Rolston KV, Tarrand JJ, Samonis G: Recent experience with Pseudomonas aeruginosa bacteremia in patients with cancer: Retrospective analysis of 245 episodes. Arch Intern Med 2000, 160(4):501-509.

17. Funada $\mathrm{H}$, Matsuda $\mathrm{T}$ : Changes in the incidence and etiological patterns of bacteremia associated with acute leukemia over a 25-year period. Intern Med 1998, 37(12):1014-1018.

18. Afessa B, Green W, Chiao J, Frederick W: Pulmonary complications of HIV infection: autopsy findings. Chest 1998, 113(5):1225-1229.

19. Nseir S, Jozefowicz E, Cavestri B, Sendid B, Di Pompeo C, Dewavrin F, Favory R, Roussel-Delvallez M, Durocher A: Impact of antifungal treatment on Candida-Pseudomonas interaction: a preliminary retrospective case-control study. Intensive Care Med 2007, 33(1):137-142.

20. Kerr JR: Suppression of fungal growth exhibited by Pseudomonas aeruginosa. J Clin Microbiol 1994, 32(2):525-527.

21. Thein ZM, Samaranayake YH, Samaranayake LP: Effect of oral bacteria on growth and survival of Candida albicans biofilms. Arch Oral Biol 2006. 51(8):672-680.

22. Lappin-Scott HM, Costerton JW: Microbial biofilms. Cambridge University Press; 1995

23. Allison DG: Community structure and Co-operation in biofilms. Cambridge University Press; 2000.

24. Pierce GE: Pseudomonas aeruginosa, Candida albicans, and devicerelated nosocomial infections: implications, trends, and potential approaches for control. J Ind Microbiol Biotechnol 2005, 32(7):309-318.

25. Senpuku H, Sogame A, Inoshita E, Tsuha Y, Miyazaki H, Hanada N: Systemic diseases in association with microbial species in oral biofilm from elderly requiring care. Gerontology 2003, 49(5):301-309.

26. Hogan DA, Vik A, Kolter R: A Pseudomonas aeruginosa quorum-sensing molecule influences Candida albicans morphology. Mol Microbiol 2004, 54(5):1212-1223.

27. El-Azizi MA, Starks SE, Khardori N: Interactions of Candida albicans with other Candida spp. and bacteria in the biofilms. J App/ Microbiol 2004 96(5):1067-1073.

28. Hogan DA, Kolter R: Pseudomonas-Candida interactions: an ecological role for virulence factors. Science 2002, 296(5576):2229-2232.

29. Kaleli I, Cevahir N, Demir M, Yildirim U, Sahin R: Anticandidal activity of Pseudomonas aeruginosa strains isolated from clinical specimens. Mycoses 2007, 50(1):74-78.

30. Grillot R, Portmann-Coffin V, Ambroise-Thomas P: Growth inhibition of pathogenic yeasts by Pseudomonas aeruginosa in-vitro: clinical implications in blood cultures. Mycoses 1994, 37(9-10):343-347.

31. Hockey LJ, Fujita NK, Gibson TR, Rotrosen D, Montgomerie JZ, Edwards JE Jr: Detection of fungemia obscured by concomitant bacteremia: invitro and in-vivo studies. J Clin Microbio/ 1982, 16(6):1080-1085.

32. Jin Y, Samaranayake LP, Samaranayake Y, Yip HK: Biofilm formation of Candida albicans is variably affected by saliva and dietary sugars. Arch Oral Biol 2004, 49(10):789-798

33. Jin Y, Zhang T, Samaranayake YH, Fang HH, Yip HK, Samaranayake LP: The use of new probes and stains for improved assessment of cell viability and extracellular polymeric substances in Candida albicans biofilms. Mycopathologia 2005, 159(3):353-360.

34. Ramage G, Vandewalle K, Wickes BL, Lopez-Ribot JL: Characteristics of biofilm formation by Candida albicans. Rev Iberoam Micol 2001, 18(4):163-170.

doi: $10.1186 / 1471-2180-10-125$

Cite this article as: Bandara et al., Pseudomonas aeruginosa inhibits in-vitro Candida biofilm development BMC Microbiology 2010, 10:125

\section{Submit your next manuscript to BioMed Central} and take full advantage of:

- Convenient online submission

- Thorough peer review

- No space constraints or color figure charges

- Immediate publication on acceptance

- Inclusion in PubMed, CAS, Scopus and Google Scholar

- Research which is freely available for redistribution

Submit your manuscript at www.biomedcentral.com/submit
C Biomed Central 